\title{
Isotopic Abundances in the Solar Corona as Inferred from ACE Measurements of Solar Energetic Particles
}

\author{
R. A. Leske*, R. A. Mewaldt*, C. M. S. Cohen*, E. R. Christian ${ }^{\dagger}$, A. C. Cummings*, \\ P. L. Slocum ${ }^{* *}$, E. C. Stone ${ }^{*}$ T. T. von Rosenvinge ${ }^{\dagger}$ and M. E. Wiedenbeck** \\ ${ }^{*}$ California Institute of Technology, Pasadena, CA 91125 USA \\ ${ }^{\dagger}$ NASA/Goddard Space Flight Center, Greenbelt, MD 20771 USA \\ ${ }^{* *}$ Jet Propulsion Laboratory, Pasadena, CA 91109 USA
}

\begin{abstract}
The isotopic composition of solar energetic particles (SEPs) has been measured using the Solar Isotope Spectrometer on the Advanced Composition Explorer. The measurements include up to 12 isotope abundance ratios for ten elements from $\mathrm{C}$ through $\mathrm{Ni}$ at energies of tens of $\mathrm{MeV} /$ nucleon in 18 large SEP events that have occurred since November 1997. These measurements clearly establish that SEP isotopic composition can vary widely (by factors of $>3$ ) from event to event, presumably due to mass fractionation processes during particle acceleration and/or transport. Elemental and isotopic abundance ratios are strongly correlated, suggesting that elemental and isotopic fractionation relative to the coronal source are largely governed by the same processes. Using empirical correlations to correct for the fractionation and obtain the coronal isotopic composition yields preliminary abundance values in good agreement with those found in the solar wind, with comparable accuracy.
\end{abstract}

\section{INTRODUCTION}

A primary goal of the Advanced Composition Explorer (ACE) mission is to better establish the elemental and isotopic composition of the Sun. Solar energetic particles (SEPs) provide a sample of solar material that may be used for such studies, but particle acceleration and transport processes can affect the arriving composition. Two distinct categories of SEP events, impulsive and gradual, are generally recognized [1]. Particles in gradual events are thought to originate as solar wind or coronal material accelerated by large shocks driven by coronal mass ejections. Elements in gradual SEP events have been measured for many years [e.g. 2]. Their abundances have been found to be highly variable from event to event but correlated with the ionic charge to mass ratio, $Q / M$ [3]. When corrected for this fractionation $[3,4]$ or averaged over many events [5], SEP abundances can be used to determine the coronal elemental composition more accurately than is possible from spectroscopic measurements for some elements such as noble gases. In principle, the coronal isotopic composition can be similarly obtained from SEPs [6, 7], which has not been possible spectroscopically for more than a few isotopes.

Unlike the case for SEP elemental measurements, before the launch of ACE there were only a few SEP heavy isotope measurements, and these included only elements up to $\mathrm{Si}$ [see, e.g. 7, and references therein]. To obtain adequate statistical accuracy, the earlier measurements sometimes required sums over several SEP events $[8,9,10]$ and the resulting values usually agreed with terrestrial abundances but with rather large uncertainties. Isolated differences were noted for some gradual events $[6,7]$, and significant enrichments of ${ }^{22} \mathrm{Ne}$ were found in ${ }^{3} \mathrm{He}$-rich periods [11].

In recent studies using ACE data, enhancements by up to a factor of $\sim 2$ were reported in the 6 November 1997 SEP event for many heavy/light isotope abundance ratios from ${ }^{13} \mathrm{C} /{ }^{12} \mathrm{C}$ to ${ }^{60} \mathrm{Ni} /{ }^{58} \mathrm{Ni}$ [12]. Using the Solar Isotope Spectrometer (SIS) on ACE, the ${ }^{22} \mathrm{Ne} /{ }^{20} \mathrm{Ne}$ ratio was observed to vary by a factor of $>3$ from event to event at energies of 24-72 MeV/nucleon [13], and similar or greater variability was found for $\mathrm{Ne}$ in other events at lower energies [14]. In the present work, we extend the previous ACE/SIS studies and present isotopic abundance measurements for $\mathrm{C}, \mathrm{O}, \mathrm{Ne}, \mathrm{Mg}, \mathrm{Si}, \mathrm{S}, \mathrm{Ar}$, $\mathrm{Ca}, \mathrm{Fe}$, and $\mathrm{Ni}$ in as many as 18 individual SEP events. The isotopic composition is highly variable, but using the abundance correlations between different species we empirically correct for the variation and obtain preliminary coronal isotopic abundances from SEPs. Observations in a subset of the events over a more limited energy interval are also reported in $[15,16]$. 


\section{OBSERVATIONS AND ANALYSIS}

Using the $\mathrm{d} E / \mathrm{d} x$ versus residual energy technique in a pair of silicon solid-state detector telescopes, the SIS instrument allows the nuclear charge, $Z$, mass, $M$, and total kinetic energy, $E$, to be determined for particles with energies of $\sim 10$ to $\sim 100 \mathrm{MeV} /$ nucleon [17]. For this study, we selected SEP events with sufficient fluxes of high energy heavy ions $(E \gtrsim 15 \mathrm{MeV} /$ nucleon, where mass resolution is best) to obtain statistically meaningful isotope abundances. Time profiles of the 18 selected events are shown in Figure 1. Their peak intensities vary by more than three orders of magnitude at these energies, with the events in the year 2000 (bottom panel) containing most of the smallest events considered for this study as well as the two largest SEP events in this solar cycle. The very high counting rates and correspondingly high rate of chance coincidences at the peaks of these two largest events severely degraded mass resolution in SIS (although the resolution is still adequate to separate elements), and therefore we restricted the isotopic analysis to the decay phases of these two events.

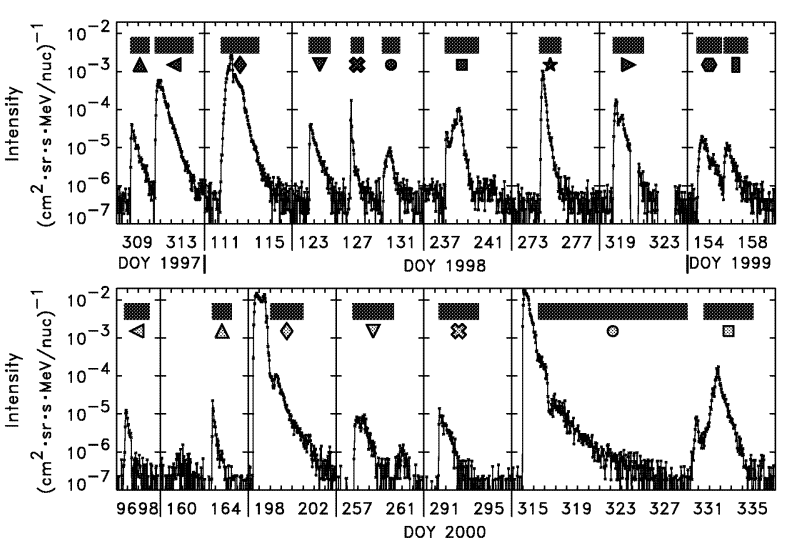

FIGURE 1. Time profiles of the 18 SEP events examined here, using hourly-averaged intensities of 21-64 MeV/nucleon oxygen from SIS on ACE. Shaded bars indicate time periods used for the isotope analysis; symbols represent these periods in Figures 3-5.

Isotopes of elements up through $\mathrm{Ni}$ are measured by SIS with a mass resolution which varies with $Z$ and $E$; for the species and energies studied here it typically ranges from $\sim 0.15$ to $\sim 0.3 \mathrm{amu}$. Details of the analysis required to obtain isotope abundance ratios and examples of mass histograms are given elsewhere $[12,13]$, but in most cases the good mass resolution makes the determination of abundances straightforward.

Obtaining coronal abundances from these data is not so simple, however, due to the fact that the SEP isotopic abundances may vary significantly from event to event [13], as shown for the ${ }^{22} \mathrm{Ne} /{ }^{20} \mathrm{Ne}$ ratio in Figure 2. Surprisingly, it appears that the composition variability was greatly reduced and nearly absent in the 1999-2000 time frame compared to that seen in 1997-1998. The reduced $\chi^{2}$ obtained from fitting a constant to the first 9 points in Figure 2 is 83 , while for the last 9 points it is a much more reasonable 1.3 , indicating a statistically significant change in the variability. Tracking the variability of the composition in future events may help to determine whether this is merely a statistical aberration or an unexplained feature of the solar cycle.

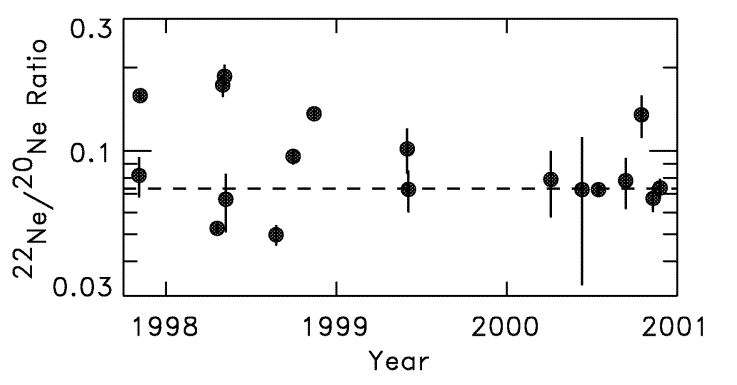

FIGURE 2. The SEP ${ }^{22} \mathrm{Ne} /{ }^{20} \mathrm{Ne}$ ratio measured by ACE/SIS at $E>15 \mathrm{MeV} /$ nucleon plotted versus the date of the event.

In obtaining coronal abundances from the highly variable SEP isotope measurements, we are guided by the experience gained in coping with a similar situation encountered for elemental abundances. The variations of heavy ion elemental abundances in individual gradual events relative to coronal values have been found to scale reasonably well as a power law in the ionic charge to mass ratio, $Q / M$ [3], with a different power law index for each SEP event. If the $Q / M$ ratio is indeed the relevant organizing parameter, then the same physical mechanism responsible for the elemental fractionation, whatever it is, should also produce variations in the isotopic abundances, since $Q / M$ will differ for two isotopes of the same element through the mass number. This implies there should be a predictable correlation between the abundances of various species, in particular between elemental and isotopic abundances. Following [6], if we base the power law fractionation index on the abundance ratio of any two reference species, such as $\mathrm{Fe} / \mathrm{O}$, $\mathrm{Na} / \mathrm{Mg}$, or, in general terms, $\mathrm{R}_{1} / \mathrm{R}_{2}$, (and remembering that $x^{\ln y}=y^{\ln x}$ ) it readily follows that we would expect the enhancement or depletion of the SEP abundance ratio for isotopes $a$ and $b$ of element $\mathrm{X}$ to be:

$$
\frac{\left({ }^{a} \mathrm{X} /{ }^{b} \mathrm{X}\right)_{\mathrm{SEP}}}{\left({ }^{a} \mathrm{X} /{ }^{b} \mathrm{X}\right)_{\text {corona }}}=\left(\frac{\left(\mathrm{R}_{1} / \mathrm{R}_{2}\right)_{\mathrm{SEP}}}{\left(\mathrm{R}_{1} / \mathrm{R}_{2}\right)_{\text {corona }}}\right)^{\frac{\ln (b / a)}{\ln \left[( Q / M ) \left(\mathrm{R}_{1} /(Q / M)_{\mathrm{R}_{2}}\right.\right.}}
$$

using the fact that $Q$ should be the same for two isotopes of the same element.

To evaluate the expected enhancement from equation (1), the ionic charge state $Q$ must be known for the reference species. Ionic charges are not often measured at SIS energies of tens of $\mathrm{MeV} /$ nucleon, and the mea- 
surements that exist show considerable variability for elements such as $\mathrm{Fe}$ [see, e.g. 18, and references therein]. Although measurements of $Q$ at lower energies might be used [3], they may not apply to SIS data since several events have been clearly shown to exhibit energydependent charge states for heavy elements $[19,20,21]$. In addition, the relevant value of $Q$ in equation (1) is that which the particles have when the elemental and isotopic fractionation takes place, which may be quite different from the value at $1 \mathrm{AU}$ if fractionation happens early and if, for example, stripping occurs in acceleration through the corona [22, 23]. Another complication not addressed in equation (1) is the fact that in gradual SEP events the abundances of elements with low first ionization potential (FIP), such as Fe, are generally enhanced over those with high FIP, such as O, by an amount which also varies from event to event $[24,25]$. In spite of the above considerations, a reasonable correlation has been shown between isotopic abundances and the $\mathrm{Fe} / \mathrm{O}$ ratio $[12,13,15]$, but uncertainties in the value of $Q(\mathrm{Fe})$ at SIS energies make it difficult to directly compare the correlation with predictions.

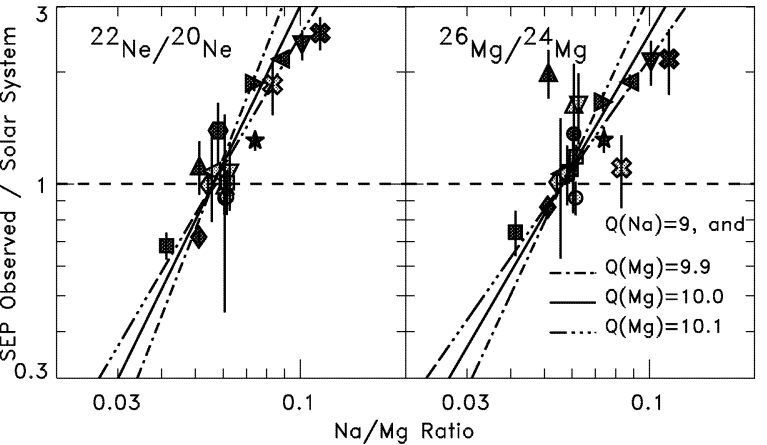

FIGURE 3. The ${ }^{22} \mathrm{Ne} /{ }^{20} \mathrm{Ne}$ (left) and ${ }^{26} \mathrm{Mg} /{ }^{24} \mathrm{Mg}$ (right) isotopic ratios versus the $\mathrm{Na} / \mathrm{Mg}$ elemental abundance ratio in each of the SEP events of Figure 1. Both isotope ratios have been normalized to their respective "solar system" value [26]. Diagonal lines show the correlations expected from equation (1), assuming the charge states of $\mathrm{Na}$ and $\mathrm{Mg}$ as indicated.

With the appropriate choice of reference species, such as $\mathrm{Na} / \mathrm{Mg}$, we do find that the isotopic and elemental abundances tend to be correlated approximately as expected from equation (1), as shown in Figure 3. Both Na and $\mathrm{Mg}$ are low-FIP elements so this ratio is unaffected by variable FIP fractionation. As pointed out by Cohen et al. [27], both elements are theoretically expected to have $\sim 2$ electrons attached over a broad range of coronal temperatures [28], and since ${ }^{23} \mathrm{Na}$ is neutron-rich with respect to ${ }^{24} \mathrm{Mg}$, there is a significant difference in $Q / M$. The solid lines in Figure 3 show the correlations expected from equation (1), assuming $Q(\mathrm{Na})=9$ and $Q(\mathrm{Mg})=10$. While this very simple model provides a good first order fit to the data, both isotope correlations appear to be shallower than expected.

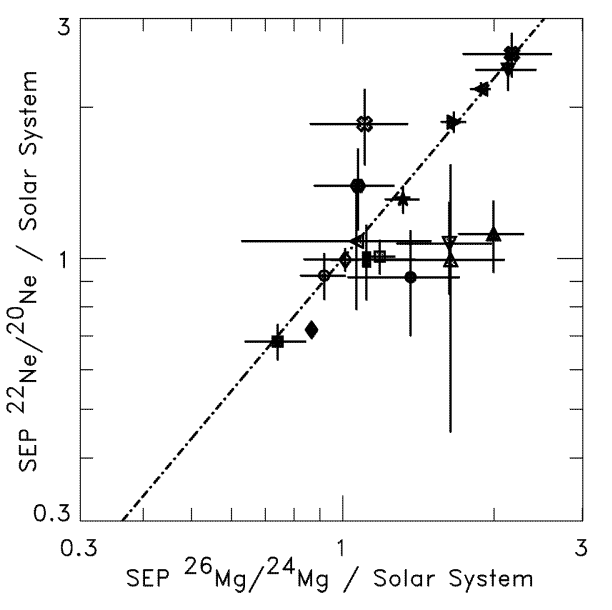

FIGURE 4. The ${ }^{22} \mathrm{Ne} /{ }^{20} \mathrm{Ne}$ versus ${ }^{26} \mathrm{Mg} /{ }^{24} \mathrm{Mg}$ isotopic ratios in each of the SEP events shown in Figure 1, normalized to standard solar system values [26]. The diagonal line shows the correlation expected using equation (1).

The predicted correlations are very sensitive to $Q$, and a small change of only $1 \%$ in the $Q(\mathrm{Na}) / Q(\mathrm{Mg})$ ratio changes the expected slope considerably, as also shown in Figure 3. Detailed equilibrium calculations [28] show that at a constant temperature $Q(\mathrm{Na}) / Q(\mathrm{Mg})$ is $>0.9$ for all temperatures from $0.5-10 \mathrm{MK}$, that is, the expected correlation should be steeper than the solid line in Figure 3 and more discrepant with the data. However, the assumption of a constant temperature for all elements is probably unwarranted. In fact, $\mathrm{Mg}$ in particular is often found to have a mean $Q$ typical of higher temperatures than most other elements $[29,30]$, which would result in a lower value of $Q(\mathrm{Na}) / Q(\mathrm{Mg})$ more consistent with the observed correlation. In any case, the relatively small amount of scatter in Figure 3 suggests that at the time of fractionation, $Q(\mathrm{Na}) / Q(\mathrm{Mg})$ does not differ by more than a few percent from event to event. This is considerably less than the event-to-event variability in individual charge states observed at $1 \mathrm{AU}$ [31] and may provide a clue as to how and when mass fractionation takes place.

If the residual scatter in Figure 3 is indeed due to variability in the $Q(\mathrm{Na}) / Q(\mathrm{Mg})$ value and if $Q$ is the same for 2 isotopes of the same element, then a better correlation should be possible using an isotope ratio as the reference value. This is illustrated by the correlation between the ${ }^{22} \mathrm{Ne} /{ }^{20} \mathrm{Ne}$ and ${ }^{26} \mathrm{Mg} /{ }^{24} \mathrm{Mg}$ ratios in Figure 4 which, for most of the events with the better-determined values, agrees very well with the expected correlation. At least no systematic deviation from the expected trend is evident. Although the outliers are small events with large uncertainties and most are not seriously discrepant statistically, it is interesting to note that they tend to lie near a value of unity on one of the two axes, as if only one isotope ratio is fractionated while the other is unaf- 


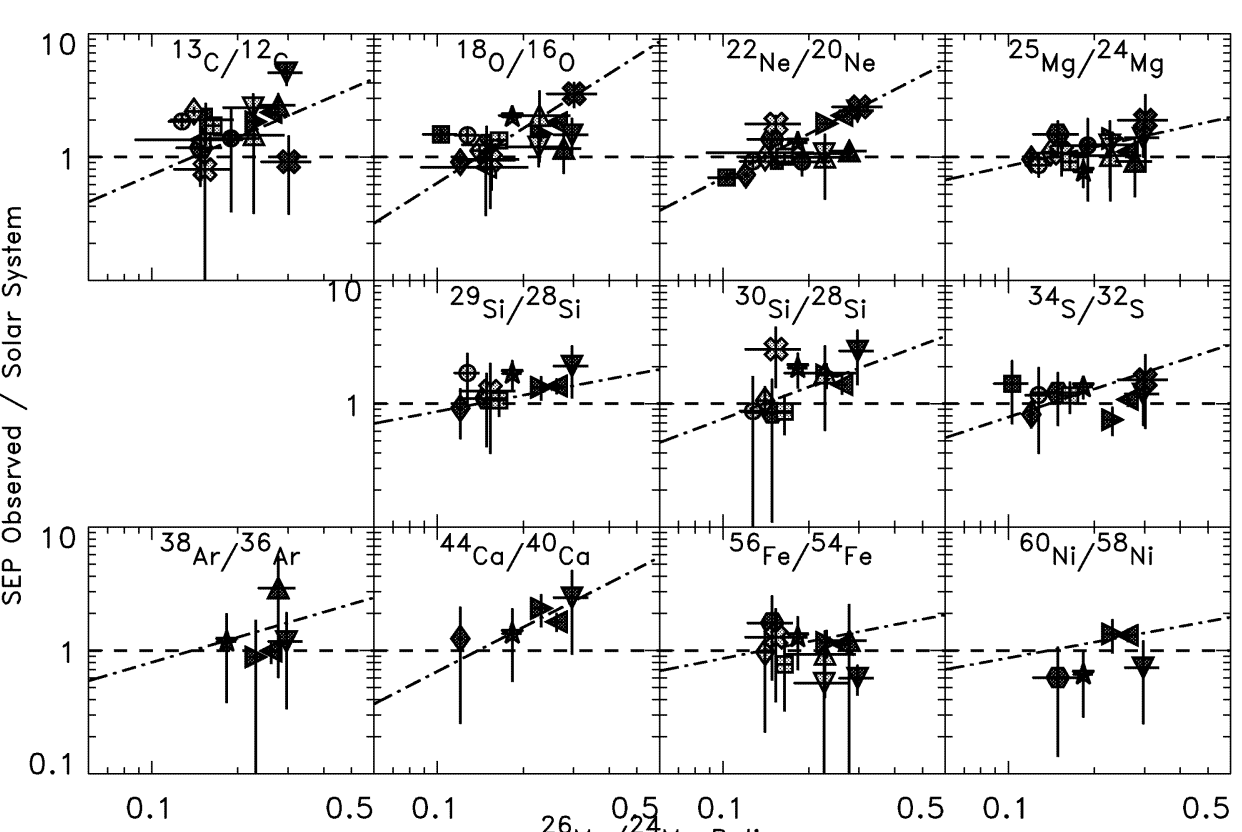

FIGURE 5. Eleven SEP isotope abundance ratios (normalized to standard abundances [26]) plotted versus the ${ }^{26} \mathrm{Mg} /{ }^{24} \mathrm{Mg}$ ratio. Symbols indicate the SEP events shown in Figure 1. The diagonal lines show the correlations expected using equation (1).

fected. Since these outlying events are among the smallest in our sample, even a small amount of contamination from impulsive events might significantly alter their composition. Of the $\sim 6$ outliers, 5 exhibit modest Fe enhancements, with $\mathrm{Fe} / \mathrm{O} \sim 0.5$. Most of the events in our study contain significant enhancements of ${ }^{3} \mathrm{He}[16,32]$, which could be due to residual material from impulsive flares resident in the interplanetary medium which is later accelerated by a shock [33]. The ion cyclotron wave resonances [34, 35] or cascading Alvén waves [36] responsible for the ${ }^{3} \mathrm{He}$ enrichment of impulsive events might also selectively enhance other species with discrete values of $Q / M$ at higher harmonics of the ${ }^{3} \mathrm{He}$ cyclotron frequency [37], and in fact significant enrichments of both ${ }^{22} \mathrm{Ne}$ and ${ }^{26} \mathrm{Mg}$ have been reported in ${ }^{3} \mathrm{He}$-rich periods $[11,38]$. If it were possible for the resonance to affect a narrow enough frequency range, perhaps only one of these two species might be enhanced, resulting in a pattern such as appears to be present in Figure 4. It will be interesting to see if this pattern persists as more events accumulate during this solar cycle.

\section{RESULTS}

The isotopic composition of $\mathrm{Ne}$ is interesting since it differs in various solar system materials. Therefore, of the 2 isotopic ratios in Figure 4, we chose the ${ }^{26} \mathrm{Mg} /{ }^{24} \mathrm{Mg}$ ratio as our abundance standard so that we can solve for the SEP Ne composition in this study. The SEP abundance values for 11 isotope ratios for elements from $\mathrm{C}$ to $\mathrm{Ni}$ are shown plotted versus this reference ratio in Figure 5. The different expected slopes arise from the different relative mass number ratios, and the data seem to follow these expected trends for certain species such as $\mathrm{Ne}, \mathrm{Mg}, \mathrm{Si}$, and $\mathrm{Ca}$. For many of the heavy elements from $\mathrm{S}$ and above, the agreement between the more limited data and the expectations is not as clear. The agreement might break down with increasing distance in $Q / M$ from $\mathrm{Mg}$ if the actual dependence on $Q / M$ is not a simple power law as we assumed. For ${ }^{13} \mathrm{C}, 12$ of the 15 data points fall above the expected correlation, including most of those for which the ${ }^{26} \mathrm{Mg} /{ }^{4} \mathrm{Mg}$ and other ratios show little or no fractionation. The reason for this is not at all clear, but if this preliminary result holds up, it suggests that ${ }^{13} \mathrm{C}$ routinely is enhanced or ${ }^{12} \mathrm{C}$ is depleted in SEP events relative to terrestrial abundances. In future work we plan to extend the isotope measurements to include ${ }^{15} \mathrm{~N}$ to see if it is similarly affected.

To correct for the $Q / M$-dependent mass fractionation, we solve equation (1) for the coronal isotope ratios. For example, using the ${ }^{26} \mathrm{Mg} /{ }^{24} \mathrm{Mg}$ ratio as the reference ratio $R_{1} / R_{2}$, the ${ }^{22} \mathrm{Ne} /{ }^{20} \mathrm{Ne}$ coronal value obtained from any SEP event would be:

$$
\left(\frac{{ }^{22} \mathrm{Ne}}{{ }^{20} \mathrm{Ne}}\right)_{\text {cor. }}=\left(\frac{{ }^{22} \mathrm{Ne}}{{ }^{20} \mathrm{Ne}}\right)_{\mathrm{SEP}} \cdot\left(\frac{\left({ }^{26} \mathrm{Mg} /{ }^{24} \mathrm{Mg}\right)_{\text {cor. }}}{\left({ }^{26} \mathrm{Mg} /{ }^{24} \mathrm{Mg}\right)_{\mathrm{SEP}}}\right)^{\frac{\ln (20 / 22)}{\ln (24 / 26)}}
$$




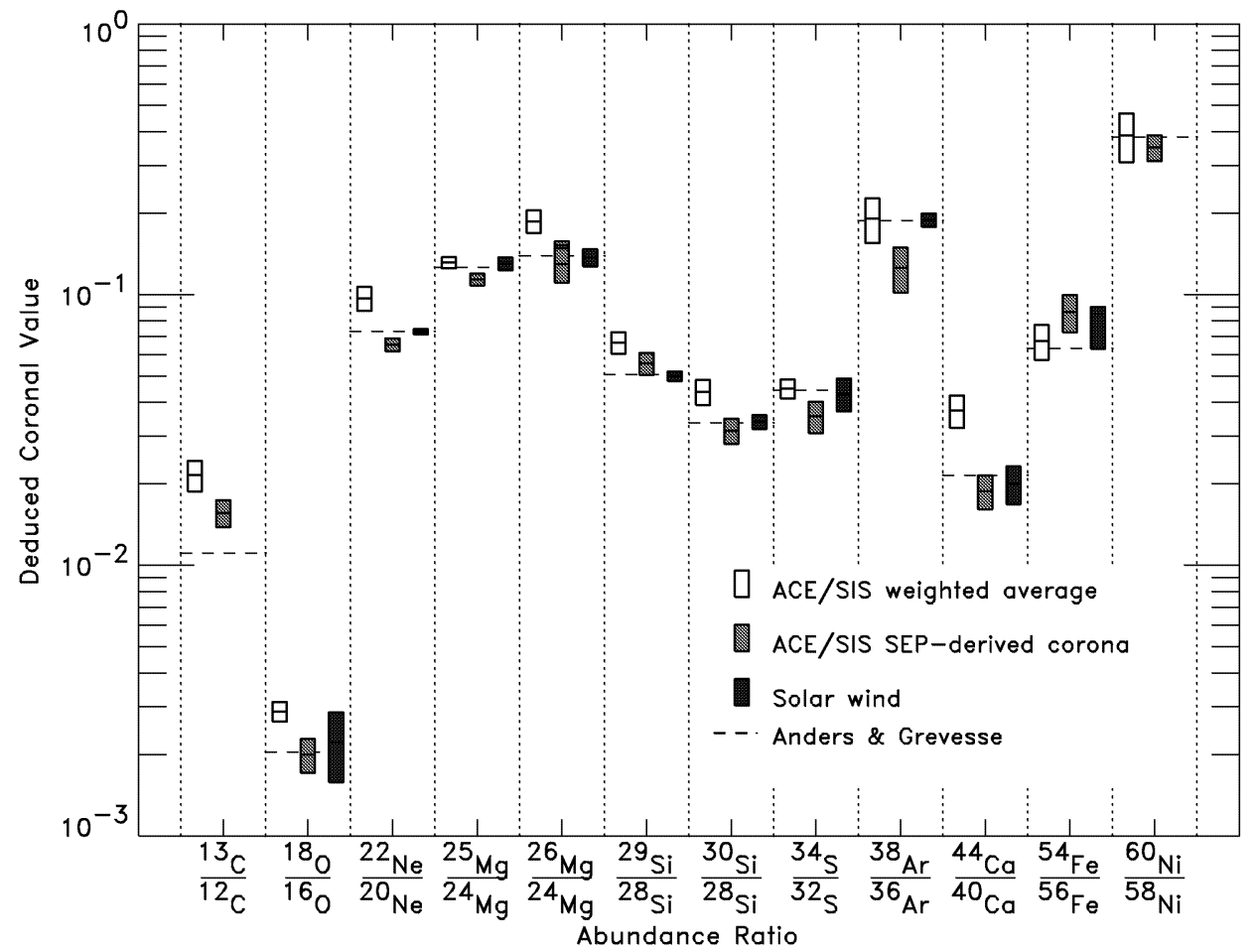

FIGURE 6. Deduced coronal source isotopic abundance ratio averages from SIS SEP measurements without correcting for fractionation (open boxes) and after correction (light grey boxes) as in equation (2). For comparison, standard solar system values (dashed lines; [26]) and measured solar wind values (dark grey boxes; [see 39, and references therein]) are shown. $\mathrm{The}{ }^{26} \mathrm{Mg} /{ }^{24} \mathrm{Mg}$ ratio served as the reference value for the fractionation corrections for everything other than the ${ }^{26} \mathrm{Mg} /{ }^{24} \mathrm{Mg}$ ratio, for which Na/Mg at the ionic charge ratios considered in Figure 3 was used.

Note that the effect of uncertainties in the selected reference value is easy to determine from this expression, the propagation of errors is straightforward, and the exponents are simple constants and do not depend on the measured values.

Preliminary solar coronal isotopic abundances obtained following the example of equation (2) and averaging over all the SIS measurements are shown in Figure 6. For comparison, we have also calculated the weighted average without correcting for the fractionation, but including in the weighting the width of the parent population distribution added in quadrature to the statistical uncertainties. This may be a more representative value for cases such as $\mathrm{S}$ or $\mathrm{Fe}$ where the data may not follow the expected fractionation correlations in Figure 5, and with a large enough data set (if unbiased by selection effects) may even average out to the coronal value as seems to be the case for elemental abundances [5]. Also, the uncorrected average is the appropriate one to consider for assessing the average arriving solar particle composition at $1 \mathrm{AU}$. For example, the uncorrected average ${ }^{22} \mathrm{Ne} /{ }^{20} \mathrm{Ne}$ ratio we find here is consistent with the value of $\sim 0.09$ of the so-called SEP component detected in lunar soils [40]. Both our corrected and uncorrected SEP values are also compared with the standard solar system values [26] and existing solar wind values [39] in Figure 6.

Although the results are preliminary, it is encouraging that this early attempt to obtain coronal abundances from the fractionated SEPs seems to yield reasonable values. With the exception of ${ }^{13} \mathrm{C}$, all of the isotope abundances are within $2.5 \sigma$ of the Anders and Grevesse [26] "solar system" values. Both the corrected noble gas isotopes ${ }^{22} \mathrm{Ne}$ and ${ }^{38} \mathrm{Ar}$ appear a bit low compared to Anders and Grevesse, but for these two species Anders and Grevesse adopted the solar wind values as their standard without accounting for mass fractionation in the solar wind of perhaps several percent [41]. So far, Ni isotope abundances have not been reported from solar wind data, so the SEP value given here is the first determination of the ${ }^{60} \mathrm{Ni} /{ }^{58} \mathrm{Ni}$ ratio in the corona.

In many cases the uncertainties on the SEP-derived coronal isotope values are comparable to those obtained from solar wind measurements. Accumulating additional SEP events will help reduce the uncertainties for the heaviest species such as $\mathrm{Ar}$ to $\mathrm{Ni}$ where there are still only a few measurements, but for most of the others a better theoretical understanding of the mass fractionation process is required to make much further progress. 
Tracking some of the puzzles uncovered here through the declining part of the solar cycle, such as the decreasing variability in recent events (Figure 2), the possible fractionation of only some isotope ratios (Figure 4), and the apparent common enhancement of ${ }^{13} \mathrm{C}$ (Figure 5) may help to shed light on the nature of the fractionation process.

\section{ACKNOWLEDGMENTS}

This research was supported by NASA at the California Institute of Technology (under grant NAG5-6912), the Jet Propulsion Laboratory, and the Goddard Space Flight Center.

\section{REFERENCES}

1. Reames, D. V., Revs. Geophys., 33, 585-589 (1995).

2. Teegarden, B. J., von Rosenvinge, T. T., and McDonald, F. B., Astrophys. J., 180, 571-581 (1973).

3. Breneman, H. H., and Stone, E. C., Astrophys. J. Lett., 299, L57-L61 (1985).

4. Garrard, T. L., and Stone, E. C., Proc. 23rd Internat. Cosmic Ray Conf. (Calgary), 3, 384-387 (1993).

5. Reames, D. V., Adv. Space Res., 15, (7)41-(7)51 (1995).

6. Mewaldt, R. A., and Stone, E. C., Astrophys. J., 337, 959-963 (1989).

7. Williams, D. L., Leske, R. A., Mewaldt, R. A., and Stone, E. C., Space Sci. Rev., 85, 379-386 (1998).

8. Dietrich, W. F., and Simpson, J. A., Astrophys. J. Lett., 231, L91-L95 (1979).

9. Dietrich, W. F., and Simpson, J. A., Astrophys. J. Lett., 245, L41-L44 (1981).

10. Simpson, J. A., Wefel, J. P., and Zamow, R., Proc. 18th Internat. Cosmic Ray Conf. (Bangalore), 10, 322-325 (1983).

11. Mason, G. M., Mazur, J. E., and Hamilton, D. C., Astrophys. J., 425, 843-848 (1994).

12. Leske, R. A., et al., Geophys. Res. Lett., 26, 153-156 (1999).

13. Leske, R. A., et al., Geophys. Res. Lett., 26, 2693-2696 (1999).

14. Dwyer, J. R., Mason, G. M., Mazur, J. E., Gold, R. E., and Krimigis, S. M., Proc. 26th Internat. Cosmic Ray Conf. (Salt Lake City), 6, 147-150 (1999).

15. Leske, R. A., et al., Proc. 26th Internat. Cosmic Ray Conf. (Salt Lake City), 6, 139-142 (1999).

16. Leske, R. A., et al., "Measurements of the Heavy-Ion Elemental and Isotopic Composition in Large Solar Energetic Particle Events from ACE", in High Energy Solar Physics: Anticipating HESSI, edited by R. Ramaty and N. Mandzhavidze, ASP Conf. Ser. 206, Astronomical Society of the Pacific, San Francisco, 2000, pp. 118-123.

17. Stone, E. C., et al., Space Sci. Rev., 86, 357-408 (1998).

18. Leske, R. A., Mewaldt, R. A., Cummings, A. C., Stone, E. C., and von Rosenvinge, T. T., "The Ionic Charge State Composition at High Energies in Large Solar Energetic Particle Events in Solar Cycle 23", in Proc.
Joint SOHO-ACE Workshop 2001, AIP Conf. Proc. , AIP, New York, 2001, this volume.

19. Mazur, J. E., Mason, G. M., Looper, M. D., Leske, R. A., and Mewaldt, R. A., Geophys. Res. Lett., 26, 173-176 (1999).

20. Möbius, E., et al., Geophys. Res. Lett., 26, 145-148 (1999).

21. Oetliker, M., et al., Astrophys. J., 477, 495-501 (1997).

22. Reames, D. V., Ng, C. K., and Tylka, A. J., Geophys. Res. Lett., 26, 3585-3588 (1999).

23. Barghouty, A. F., and Mewaldt, R. A., Astrophys. J. Lett., 520, L127-L130 (1999).

24. Garrard, T. L., and Stone, E. C., Adv. Space Res., 14, (10)589-(10)598 (1994).

25. Mewaldt, R. A., et al., "Variable Fractionation of Solar Energetic Particles According to First Ionization Potential", in Acceleration and Transport of Energetic Particles Observed in the Heliosphere: ACE 2000 Symposium, edited by R. A. Mewaldt et al., AIP Conf. Proc. 528, AIP, New York, 2000, pp. 123-126.

26. Anders, E., and Grevesse, N., Geochim. Cosmochim. Acta, 53, 197-214 (1989).

27. Cohen, C. M. S., et al., Geophys. Res. Lett., 26, 2697-2700 (1999).

28. Arnaud, M., and Rothenflug, R., Astron. Astrophys. Suppl., 60, 425-457 (1985).

29. Luhn, A., et al., Proc. 19th Internat. Cosmic Ray Conf. (La Jolla), 4, 241-244 (1985).

30. Leske, R. A., Cummings, J. R., Mewaldt, R. A., Stone, E. C., and von Rosenvinge, T. T., Astrophys. J. Lett., 452, L149-L152 (1995).

31. Möbius, E., et al., "Survey of Ionic Charge States of Solar Energetic Particle Events During the First Year of ACE", in Acceleration and Transport of Energetic Particles Observed in the Heliosphere: ACE 2000 Symposium, edited by R. A. Mewaldt et al., AIP Conf. Proc. 528, AIP, New York, 2000, pp. 131-134.

32. Wiedenbeck, M. E., et al., "Enhanced Abundances of ${ }^{3} \mathrm{He}$ in Large Solar Energetic Particle Events", in Acceleration and Transport of Energetic Particles Observed in the Heliosphere: ACE 2000 Symposium, edited by R. A. Mewaldt et al., AIP Conf. Proc. 528, AIP, New York, 2000, pp. 107-110.

33. Mason, G. M., Mazur, J. E., and Dwyer, J. E., Astrophys. J. Lett., 525, L133-L136 (1999).

34. Fisk, L. A., Astrophys. J., 224, 1048-1055 (1978).

35. Temerin, M., and Roth, I., Astrophys. J. Lett., 391, L105-L108 (1992).

36. Miller, J. A., Space Sci. Rev., 86, 79-105 (1998).

37. Bochsler, P., and Kallenbach, R., Meteoritics, 29, 653-658 (1994).

38. Slocum, P. L., et al., "Measurements of Heavy Elements and Isotopes in Small Solar Energetic Particle Events", in Proc. Joint SOHO-ACE Workshop 2001, AIP Conf. Proc., AIP, New York, 2001, this volume.

39. Wimmer-Schweingruber, R. F., Bochsler, P., and Wurz, P., "Isotopes in the Solar Wind: New Results from ACE, SOHO, and WIND", in Solar Wind Nine, edited by S. R. Habbal et al., AIP Conf. Proc. 471, AIP, New York, 1999, pp. 147-152.

40. Wieler, R., Space Sci. Rev., 85, 303-314 (1998).

41. Kallenbach, R., et al., Space Sci. Rev., 85, 357-370 (1998). 